\title{
A Case History in Interactive Problem-Solving
}

\author{
Richard J. Fateman \\ Department of Mathematics \\ Maseachusetts Institute of Technology \\ Cambridge, Mass.
}

\section{ABSTRACT}

MACSYMA [1], a computer program for algebraic manipulation, is used to solve, symbolically and exactly. a problem involving integration and the solution of an infinite set of linear equations. This is a tutorial in the use of MACSYMA, and illustrates some of the choices available to the interactive user which aid in solving a mathematical problem.

$$
\text { I - The problem }
$$

In [2], H. P. Greenspan develops a model of the generation of large scale magnetic fields from small scale motions of a conducting fluid. We take as a starting point, a set of equations (whose significance will not be discussed here) to be solved for coefficiente $B[2 N+1]$. The equations are

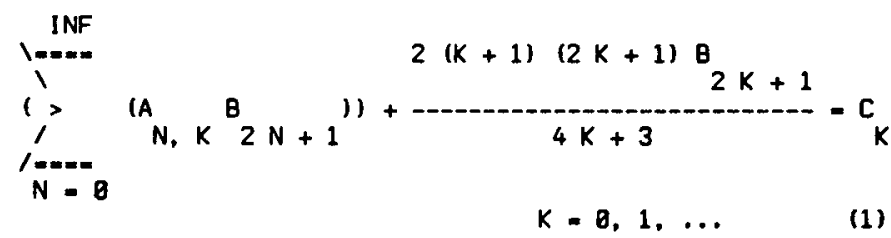

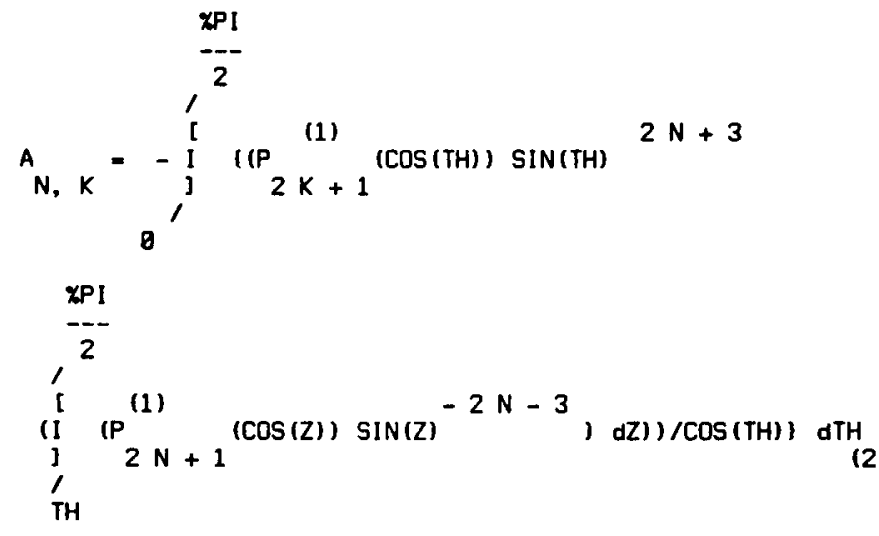

$$
\begin{aligned}
& \text { and }
\end{aligned}
$$

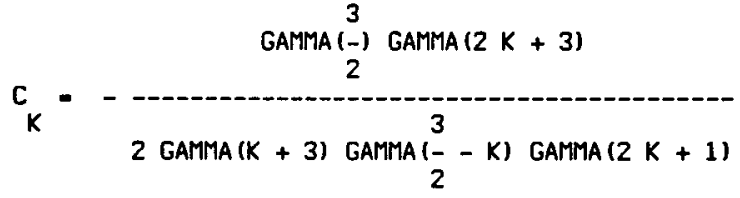

where $P$ is an associated Legendre function. The above displays were generated by MACSYMA, and includes a summation sign and two integrals (stand back a bit if you can't see them).

$$
\text { II - The Solution }
$$

Equation (1) is an infinite set of linear equations. The technique for solving for the $B[i]$ is to generate and solve the first equation for $B[1]$, then generate the next equation, and solve these two equations together to get $B$ [1] and $B[3]$, etc. One hopes that the sequence of values for $B$ [1] derived in this way converges, and similarly for $B$ [3]. etc. It is also expected, in the context of this problem. that $B[i]$ tends to $B$ for large $i$.

We will assume that the reader has a slight acquaintance with MACSYMA [1] or a similar system for algebraic manipulation.
Let us do the easy parts first. Equation (3) defines a set of values for $C$. We could define a function $C(K)$ which when given the value for $K$ returns the value for $C(K)$, but this would repeatedly compute $C$. We might as well save them in an array, especially since in MACSYMA it is just as simple. In this case, when $[$ [1] is requested the first time, it gets computed using the formula, and is then stored in memory so that subsequent references to $C[1]$ are just read out of storage. The definition (and display) of $C$ as given to MACSYMA is:

(C1) $C[K]:=-$ GAMMA $(3 / 2) \because G A M M A(2 * K+3) /$

$$
\text { (2\%GAMMA }(2 * K+1) \text { \%GAMMA }(3 / 2-K) ; G A M M A(3+K)) \text {; }
$$

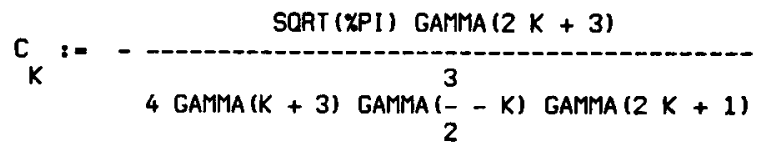

Note that MACSYMA has simplified the GAMMA(3/2) Let us compute a list of the first 4 values.

(C2) $[C[\theta], C[1], C[2], C[3]]$;

(D2)

$$
\left[\begin{array}{cccc}
1 & 1 & 5 & 7 \\
-\frac{-}{2} & -\frac{-}{2}, & \frac{-}{32}, & \left.-\frac{--}{80}\right]
\end{array}\right.
$$

Next, let us compute the assoclated Legendre functions by any convenient method. Rodrigues' formula is handy:

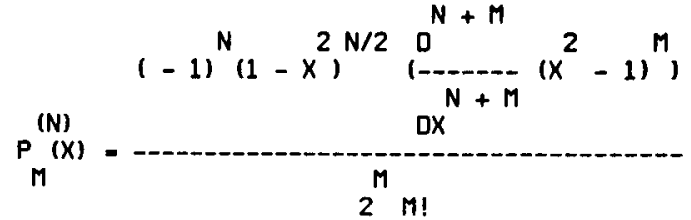

But since all we need is the $N=1$ case evaluated at $\cos (y)$. we can replace $(1-X \uparrow 2) \uparrow(N / 2)$ by $\sin (y)$, and after taking derivatives with respect to $x$, substitute cos(y) for $x$. We can set up an array of functions, one for each associated Legendre function at a cosine point by

(C3) ALF [M] (Y) : $=-\operatorname{SIN}(Y) /(2 \uparrow M r d Y$ !) rSUBST (COS $(Y), X$ $\operatorname{DIFF}((X \uparrow 2-1) \uparrow M, X, M+1))$

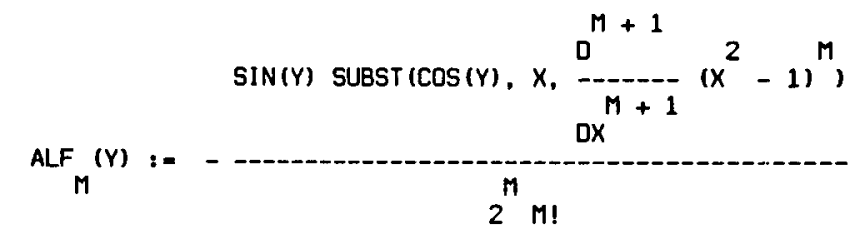

(C4) ALF [5] (X);

$(04)-\left(1 / 200\left(\cos ^{2}(x)-1\right)^{2}+57600 \cos ^{4}(x)+86400 \cos ^{2}(x)\right.$

$(\operatorname{Cos}(x)-1)) \sin (x)) / 384 \theta$

This might be sufficient, but it is somewhat neater to convert these results into a simpler form involving just sines or just cosines. Since formula (2) already involves sines, we choose sines. We can do this by defining yet another array. which has the same values as ALF, but with $(1-\operatorname{SIN}(Y) \uparrow 2)$ substituted for $\operatorname{COS}(Y) \uparrow 2$ everywhere. Since we also want to convert $\operatorname{COS}(Y) \uparrow 4$ (etc) to $\operatorname{SIN}^{\prime} s$, we use RATSUBST:

(C5) ALF1 [M] (Y) : - RATSUBST $(1-\operatorname{SIN}(Y) \uparrow 2, \operatorname{COS}(Y) \uparrow 2, \operatorname{ALF}[M](Y))$ 
It ceeme.preferable to divide the denominator through, co we types

(C6) RATEXPANITS

(C7) ALF1 [5] (X),

(07)

$$
\frac{315 \sin ^{5}(x)}{8}+\frac{185 \sin ^{3}(x)}{2}-15 \sin (x)
$$

The next problen is to evaluate the integrals of equation (2). We may have no a priori knowledge of whether the integral is eingular, or if it can be done exactly by conventional methods, or if it involves epecial functions. We can let MACSYMA take care of these problems if we just defines

(C8) A $[N, K]:=$ INTEGRATE $(-\operatorname{SIN}(T H) \uparrow(2 * N+3) / \operatorname{COS}(T H)$ * ALF1 [2*K+1] (TH) *

INTEGRATE (ALF1 [2:N+1] $(Z) / \operatorname{SIN}(Z) \uparrow(2 x N+3), Z, T H, X P I / 2)$, $\mathrm{TH}, \mathbf{0}, \mathrm{XPI} / 2)$

This is a time-consuming calculation since definite integration in MACSYMA is done with great concern for a function's behavior, and can use contour integration algor ithms, and various heuristic methods. Another fault with this formulation is that if we are going to compute many of these $A[N, K]$, we will be recomputing the inner integral. Which depends only on $N$, repeatedly. Although this formulation can be used, it is helpful to analyze the problem fur ther.

Some experimentation shows that the inner integral is alwaye 8 at XPI/2, and that all we have to do is compute:

(C9) INNERINT [N] : : -INTEGRATE (ALF1 $[2 * N+1](T H) / S I N(T H) \uparrow(2 x N+3), T H)$

An improvewent in running time can be made by first cancelling the SIN's in the denominator and expanding the integrand. After this expansion, the integration program produces an integral entirely in terms of 1/TAN (TH).

Wi thout this transformation, the integration program returns a much more unwieldy answer. The interactive nature of MACSYMA aided in this discovery, because both of the

indicated programs were tricc out.

(C19) INNERINT [N] : - -INTEGRATE (RATEXPAND (ALF1 [2 $N+1]$ (TH) / $\operatorname{SIN}(T H)+(2+N+3)), T H)$ s

(C11) INNERINT [2] ,

(011)

$\frac{15}{8 \text { TAN (TH) }}+\frac{15}{3}$ TAN $^{\text {TTH }}$ TAN $^{\text {TTH }}$

This is fairly neat. What we would really profer, is to remove the TAN' 8 and put in SIN's, or at least SIN's and COS's. This can be done quite dimply using RATSUBST.

(C12) I1 [N] : =RATSUBST (1-SIN (TH) $22, \operatorname{COS}(T H) \uparrow 2$ RATSUBST (SIN (TH) /COS (TH), TAN (TH), INNERINT [N] ) ) 8

(C13) 11 [2]

(D13)

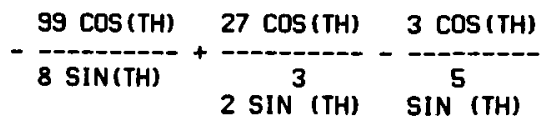

Now we are at the point where we can generate the integrand for the outer integral, and examine what it is we really are after. The integrands have a suprisingly oimple structure.

(C14) $R(N, K):=R A T E X P A N D(-S I N(T H) \uparrow(2 w N+3) /$

COS (TH) *ALF]. [2*K+1] (TH) *II [N] ) 8

(C15) $R(0,1)$

(015) 5 $\frac{15 \operatorname{SIN}^{5}(T H)}{2}-6 \operatorname{SIN}^{3}(T H)$

(C16) $R(1,0)$

(016)
(C17) ' $R(1,1)$,

(017)

$\frac{105 \operatorname{SIN}^{7}(T H)}{4}{ }^{5}(T H)-12 \sin ^{3}(T H)$

It can bo determined that in fact all integrands are sums of powers of SIN (TH), and only odd powers, at that.

Integration could be done by using the INTEGRATE command, but this computation would proceed by doing the indefinite integral, and then return the difference of ovaluating at the limite. This is fine for one or two cases, but we can save considerable time by computing definite integrals of powere of SIN, and etoring them in an array. We can also illustrate some more facilites of MACSYMA along the Hay.

There is a little formula available for computing the integral from $\theta$ to XPI/2 of an odd power of SIN. It is:

(C18) ISIN [N] : $=B L D C K([M], M:(N-1) / 2$, RETURN (2^ $(2 * M) *(M !) \uparrow 2 / N !))$ s

Now we are ready to produce the $A[N, K]$ :

(C19) A [N, K] : - BLDCK ( [EXP, ANS, COEF, H] .

ANS: 8 ,

$\operatorname{EXP:R(N,K)}$

$H: H I P O W(E X P, S I N(T H))$

FOR I:H STEP -2 THRU 1 DO

ICOEF : BOTHCOEF (EXP, SIN (TH) $\uparrow I)$,

ANS: ANS+ISIN [1] *INPART (COEF, 1 ),

EXP: INPART (COEF, 2)],

RETURN (ANS) ) 8

Let us describe the steps in the program above:

(line 1) the variables EXP, ANS, COEF, and $H$ are declared local to the BLOCK. That is, values of EXP (etc.) within this program bear no relation to values previously assigned to EXP (etc.).

(line 2) Set ANS to 8 . ANS will have the answer when we conclude execution of this program.

(I ine 3) Set EXP to the integrand computed by function $R$.

(line 4) Set $H$ to the highest power of SIN (TH) in EXP.

(line 5) Set up a loop on I for all (odd) powers of SIN (TH) in EXP.

(line 6) Set COEF to a list of (1) the coefficient of SIN (TH) $\uparrow I$ in EXP, and (2), the rest of EXP with the SIN (TH) I I term removed.

(line 7) Add to ANS the iniegral of SIN(TH) $\uparrow$ I times the appropriate coefficient. (INPART (COEF, 1) picks out the firet part of the answer from BOTHCOEF. I

(line 8) Set EXP to the part which remains after removing

the SIN(TH) $\uparrow$ I term from the integrand. (Also, return to I ine 6 for the next value of $($ unless $(=1)$

(line 9) Return ANS as the value of the integral, and set $A[N, K]$ to that value.

We can now set up the equations to be solved. We can convert equation (2) to the form

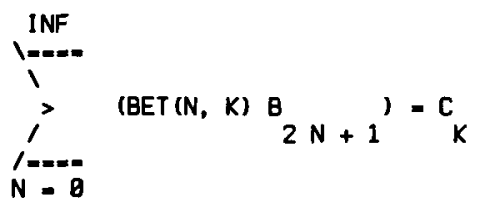

where $B E T(N, K)$ is $A[N, K]$ if $N$ is not equal to $K$, otherwise to $A[N, K]$ + an additional term. It looks like this:

(C20) BET $(N, K):-$ IF (N\#K) THEN A [N,K] ELSE

$A[N, K]+(2 x N+1) *(2 r N+2) /(4 x+N+3) s$

SETUP sets up $N$ equations, EQ [0] , ... EQ $[N-1]$.

(C21) SETUP (N) : = FOR L:B THRU $N-1$ DO

EQ [L] : SUM $(B E T(1, L) * B[2 * I+1], I, 1, N)=C[L]$

Finally we define a program which sets up lists of equations and variables, and calls the built-in command, SOLVE. CONS is a progran which adds an element to the beginning of a list. An empty list is $[$. 
(C22) ISOLVE (N) : = BLOCK ( [EQS, VARS],

EOS: [], VARS: I],

SETUP (N)

FOR L: 1 THRU $N$ DO

[EQS: CONS (EQ [L-1], EQS)

VARS: CONS (B [2 iL +1] , VARS)]

RETURN (SOL VE (EOS, VARS) ) ) \&

Let's try it out.

(C23) ISOLVE (1);

(023)

[E23]

This is a list of the solutions from SOLVE. Evaluate its

(C24) EV (\%)

(024)

$$
{ }_{3}=-\frac{15}{16}
$$

If we set TIME to TRUE, we can find out how long computations take.

(C25) TIME: TRUE

TIME $=4$ MSEC.

(C26) ISOLVE (2);

TIME= 11853 MSEC.

(D27)

[E26, E27]

(C28) EV (X) :

TIME = 11 MSEC.

$$
{ }_{5}=\frac{35}{144}, B_{3}=-\frac{35}{48}
$$

(C29) ISOLVE (3);

TIME- 51849 MSEC

(031)

$[E 29, E 39, E 31]$

(C32) EV(X);

TIME= 14 MSEC.

(032)

$$
\mathrm{CB}_{7}=-\frac{63}{512} \cdot \mathrm{B}_{5} \cdot \frac{77}{576}, \mathrm{~B}_{3}=-\frac{35}{48}
$$

In fact, we can run this example out much fur ther, and we note that we have an exact solution from ISOLVE (N) for the terms up to $2 \mathrm{~N}-1$. That is, the infinite set of equations has an exact solution. This surprising situation is a result of the fact that $A[N, K]$ is $B$ for $K>N$, which is obvious if we look at the values of the $A[N, K]$. We can display them in a matrix.

First we have to offeet the values of $A$ by 1 , since matrices in MACSYMA (as in most other places) begin their indexing at 1 , not 8 . We can do it as follows:

(C33) $A A[N, K]:-A[N-1, K-1] 8$

TIME- 20 MSEC.

(C34) GENMATRIX $(A A, 3,3)$;

TIME = 3955 MSEC.

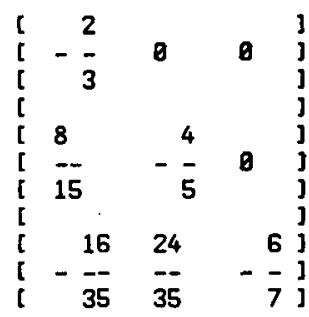

The surprising pattern in the values of the double integral of equation (2) led to a recursion relation (note that $A A[1,2]=A A[1,1]$ wAA $[2,2]$, etc.) and a formula to evaluate it analytically for all values of $N$ and $K$. Fur ther examination shows that the matrix "BET" leads to a system where each row can be cancelled, except for the diagonal term, by a multiple of the next. This diagonal system was explicitly solved to yield a closed form result for the $B(2 N+1]$. For some details of this, consult the paper by Greenspan [2].

\section{III - Additional Comments}

As is fairly typical in mathematics, the problem as originally stated (and which was, in fact, also solved) was of no interest because the equations were erroneous. In fact, that use of MACSYMA was somewhat more elegant, because an approximation to a non-rational portion of the integrals was computed using power series. However, the values of $B[2 N+1]$ which were produced by that formulation did not converge, but oscillated and grew. This unsatisfactory result suggested that MACSYMA was being asked the wrong questions, as indeed was the case. A corrected version of the equations leads, as we have seen, to a decreasing and convergent series.

$$
\text { IV - Lonclusions }
$$

We would like to briefly point out a few of the facilities in MACSYMA which make it useful to a working applied mathematician.

11) Incorrect and/or useless formulations can be discarded more rapidly by following such formulations through to symbolic results (or numerical resulto) in sufficient quantity to answer basic questions concerning the behavior of a solution.

(2) Partial results can be examined immediately to determine procedures to be followed in the next steps.

(3) Large numbers of techniques can be drawn upon, without explicit user definition. For example, the presence of GAMMA function evaluation and simplification routines, the INTEGRATE command, the SOLVE command.

(4) The ability to set up hierarchies of programs which duplicate the sequence of typed in commands, once a sequence is, in fact, well-defined, makes it easy to initiate complicated calculations in a simple fashion. Furthermore, the extension to more terms or higher accuracy is usually extremely simple.

\section{Acknowledgments}

Work reported herein was supported in part by Project MAC, an M.I.T. interdepartmental laboratory sponsored by the Advanced Research Projects Agency, Department of Defense, under Office of Naval Research Contract No014-7B-A-B362-BO1 and in part by the Department of Mathematics, M.1.T. Under National Science Foundation Grant GP 22796.

I would like to thank H. P. Greenspan for introducing me to this problem, and $M$. l sraeli for his suggestions concerning various modifications of this problem and its solution.

\section{References}

[1] Bogen, R., et al, The MACSYMA Manual, Project MAC, M.I.T.

[2] Greenspan, H. P., "On A'pha-dynamos", J. of Appl ied Math. (to appear). 